\title{
Um Mecanismo de Incentivo à Cooperação para Distribuição de Vídeo P2P com Protocolo Push/Pull
}

\author{
José Antonio Oliveira, Felipe Prado, Flávia M. de Lima, Alexandre Sztajnberg
}

Resumo - Redes P2P para distribuição de vídeo podem ser prejudicadas por nós com comportamento não cooperativo. Neste artigo o comportamento de nós não cooperativos é modelado e seus efeitos avaliados em uma rede P2P Push/Pull para distribuição de vídeo. A partir desta avaliação, um mecanismo de seleção dinâmica de vizinhos baseado na reputação dos nós é proposto e também avaliado. Este mecanismo procura melhorar a qualidade da vizinhança de nós cooperativos e penalizar nós não cooperativos com uma vizinhança de nós igualmente não cooperativos a fim de incentivá-los a mudar seu comportamento.

Palavras-Chave - P2P, difusão de vídeo, nós não cooperativos.

Abstract - Uncooperative nodes can degrade video distribution in P2P networks. In this paper the behavior of uncooperative nodes is modeled and their effect evaluated on P2P networks that use the Push/Pull protocol to distribute video streams. Based on this evaluation, a dynamic neighbor selection mechanism, which uses reputation information, is also proposed and evaluated. This mechanism aims to improve neighborhood quality for cooperative nodes connecting them to equally cooperative nodes while imposing a penalty to uncooperative nodes as a way to encourage a change on their behavior.

Keywords - P2P, video transmission, uncooperative nodes.

\section{INTRODUÇÃO}

Redes Par-a-Par, ou Peer-to-Peer (P2P), vêm sendo consideradas como opção para a distribuição de vídeo na Internet, devido às suas características de escalabilidade e distribuição de responsabilidades. Os nós integrantes da rede P2P formam uma rede sobreposta para distribuir e encaminhar pedaços de vídeo de forma cooperativa, contribuindo com recursos de processamento e canais de comunicação, com isto diminuindo a necessidade de canais com grande largura de banda e servidores com alta disponibilidade de recursos.

A distribuição de vídeo em redes P2P também apresenta desafios, pois é frequente que alguns nós, geralmente com mais recursos que os demais, fiquem sobrecarregados porque nem todos os participantes estão dispostos a cooperar. Com a existência de muitos nós com comportamento não cooperativo, as redes $\mathrm{P} 2 \mathrm{P}$ passam a apresentar características de um sistema centralizado. Na distribuição de vídeo este comportamento tende a ser ainda mais nocivo, uma vez que os usuários não estão em busca apenas da disponibilidade do arquivo contendo o vídeo, mas também esperam qualidade na sua exibição.

Neste trabalho é apresentada uma avaliação do impacto de nós não cooperativos, com diferentes gradações, em redes $\mathrm{P} 2 \mathrm{P}$ na distribuição de fluxos de vídeo que utilizam o protocolo Push/Pull como método de difusão, proposto em [1]. A partir desta avaliação é proposto e avaliado um mecanismo de seleção de vizinhos, baseado em uma forma simples de reputação, para incentivar a cooperação.
Verificou-se que o mecanismo proposto ajuda a agrupar nós cooperativos, aumentando a qualidade de vídeo recebida por estes. Percebeu-se também alguma penalidade imposta aos nós não cooperativos devido ao agrupamento, como, por exemplo, o aumento do tempo total de finalização do vídeo, indicando que o mecanismo incentiva nós que cooperam e pune os nós que não cooperam.

O modelo e o mecanismo proposto foram implementados e avaliados no simulador PeerSim com o módulo P4S para o protocolo Push/Pull. Em uma rede real, o mecanismo de seleção dinâmica de vizinhos pode ser implementado utilizando o modelo arquitetural adotado por redes P2P privadas para compartilhamento de arquivos, que agrega informações sobre a participação dos nós em seus supernós.

A Seção II discute as características do protocolo Push/Pull e a Seção III os trabalhos relacionados. A Seção IV apresenta o ambiente de simulação e as métricas propostas para as avaliações. A Seção V analisa o impacto causado por nós não cooperativos na rede. A proposta do mecanismo de seleção de vizinhos é apresentada na Seção VI e a análise de seus resultados na Seção VII. A Seção VIII conclui o trabalho.

\section{CONCEITOS BÁSICOS}

A transmissão de arquivos e fluxos de vídeo em redes $\mathrm{P} 2 \mathrm{P}$ geralmente requer a divisão do conteúdo em pedaços e a transmissão dos mesmos de forma independente através da rede. Este é o caso do BitTorrent [3], para compartilhamento de arquivos e, do CoolStreaming [14], para a transmissão de fluxos de vídeo.

\section{A. Modelos de difusão de pedaços em redes $P 2 P$}

Para sistemas que utilizam a divisão em pedaços para a transmissão de conteúdo, o mecanismo usado na difusão destes pedaços entre os nós é uma questão importante. Três modelos se destacam: push, pull, e o modelo baseado em estado do nó. Além destes, existem modelos híbridos, que mesclam características de alguns dos três modelos mencionados.

No modelo push, os pedaços são enviados de um nó pai para nós filhos proativamente. O modelo push é indicado para redes estruturadas, com topologia baseada em árvore [13].

No modelo pull um nó filho faz uma requisição de um pedaço ao nó pai sem saber se ele o possui. Aqui não ocorre o problema de duplicação como no modelo push (caso um nó tenha dois pais), porém, pode ocorrer starvation: um filho pode nunca encontrar um nó pai que possua o pedaço que ele deseja. Em geral, o modelo pull está associado a sistemas não estruturados, onde um nó filho pode ser suprido por vários nós pai, diminuindo assim a possibilidade de ocorrência de starvation. Na prática, sistemas que utilizam exclusivamente o modelo pull em geral estão associados a um mecanismo de troca de informações. 
Em modelos baseados em estado [14], cada nó mantém uma lista dos pedaços que possui e uma lista dos pedaços que estão na eminência de serem reproduzidos. Os nós fazem a troca destas listas com os seus vizinhos e, desta forma, tornam possível que os nós tenham informações sobre o estado dos outros, permitindo a requisição de pedaços específicos.

Modelos híbridos para a distribuição de pedaços, como o Interleave [10], combinam os modelos push e pull, e adicionam uma política de seleção de pedaços que dispensa a troca de informações entre os nós. O Interleave, também denominado protocolo Push/Pull, foi proposto para transferência de arquivos, porém [1] mostra a viabilidade do uso deste modelo para distribuição de vídeo.

\section{B. O modelo de difusão de pedaços Push/Pull}

$\mathrm{O}$ modelo de rede $\mathrm{P} 2 \mathrm{P}$ para distribuição de vídeo proposto em [1] possui uma única fonte, que divide o conteúdo do vídeo em pedaços para serem distribuídos entre os nós da rede sobreposta de forma independente. A rede considerada é do tipo não estruturada, com topologia em malha e simétrica. Os pedaços são gerados a uma taxa constante pela fonte e cada pedaço possui um identificador único relacionado com a sua ordem de criação.

Um nó é considerado ativo a partir da primeira recepção de um pedaço de vídeo. Ao ser ativado, este torna-se capaz de iniciar a execução do protocolo Push/Pull, enviando e pedindo pedaços do vídeo à seus vizinhos. Um nó não ativado permanece como um nó passivo. Cada nó $n$ da rede possui uma lista com a referência para todos os seus vizinhos, podendo contatar apenas estes nós. Durante a operação da rede, após ser ativado, cada nó alterna seu estado entre os modos push e pull. O nó fonte permanece sempre no estado push.

No estado push, um nó $n$ seleciona um vizinho $v$ aleatoriamente, e um pedaço $k$ entre os diversos pedaços recebidos; em seguida envia uma mensagem a $v$ questionando se este deseja $k$. Se $v$ não possui $k$ e tiver largura de banda para download disponível, este envia uma mensagem a $n$ aceitando a oferta. $\mathrm{O}$ nó $n$ envia o pedaço $k$ ao nó $v$. Caso contrário, $v$ envia uma mensagem a $n$ declinando a oferta.

No estado pull, um nó $n$ envia uma mensagem de requisição de pedaço $k$ a um nó vizinho $v$ aleatório. Se $v$ possuir o pedaço $k$ e tiver largura de banda de upload disponível, este aceita a requisição e em seguida envia o pedaço ao nó $n$. Caso contrário, o nó $v$ rejeita a requisição.

Um nó alterna seu estado depois de uma requisição ser aceita e o pedaço correspondente ser transferido, ou depois de receber um número máximo de negações às suas requisições. $\mathrm{O}$ tempo gasto nos estados push ou pull depende do sucesso das requisições e da disponibilidade de banda, tanto do vizinho quanto do nó requisitante.

Políticas inteligentes para selecionar os pedaços, como o mais raro primeiro, não podem ser implementadas diretamente no modelo Push/Pull pois seria necessário o conhecimento dos pedaços que outros nós possuem. O modelo sugerido por [1] adota a mesma política empregada em [10], que permite preencher lacunas na sequência de pedaços sem a necessidade de troca de tabelas de pedaços entre os nós:

No estado push, o nó $n$ envia o pedaço com o maior identificador entre os diversos pedaços recebidos via estado push de seus vizinhos;

No estado pull, o nó $n$ requisita o pedaço com o menor identificador que não possua. Este estado tende a acontecer menos frequentemente que o estado push, mas é eficaz para permitir o recebimento de todo os pedaços.

\section{TRABALHOS RELACIONADOS}

$\mathrm{O}$ uso de redes P2P para distribuição de vídeo e mecanismos de incentivo são abordados em [2] [5] [14] com diversos níveis de tratamento.

Mais próximo de nosso trabalho, [1] fez um estudo do protocolo Push/Pull com a finalidade de avaliar o comportamento e desempenho do mesmo na tarefa de distribuição de fluxo de vídeo na Internet combinando os modelos push e pull. Os resultados foram comparados aos obtidos em [10], que propôs o protocolo Interleave e adotou a política de seleção de pedaços descrita na seção anterior.

Estudos têm mostrado que nós que atuam como parasitas, consumindo recursos sem contribuir, são maioria em redes $\mathrm{P} 2 \mathrm{P}$ para compartilhamento de arquivos [11]. Além disso, usuários mal intencionados e que trapaceiam para obter vantagens podem degradar os serviços oferecidos pelas redes $\mathrm{P} 2 \mathrm{P}$.

Em [8], e nos surveys apresentados em [12] e [15] são identificados os tipos de comportamentos maliciosos e não colaborativos em redes $\mathrm{P} 2 \mathrm{P}$ para distribuição de fluxos de mídia. Os mecanismos de incentivo à cooperação propostos nestas redes são avaliados e a eficácia dos mesmos para tornar as redes mais robustas a nós maliciosos e não cooperativos é comparada. Algumas das redes avaliadas nestes trabalhos utilizam modelos híbridos push-pull. Por exemplo, Chainsaw, PRIME, e BAR Gossip, embora considerem diminuir a frequência da troca de tabelas de pedaços, possuem características diferentes do Interleave e apenas o BAR Gossip trata de nós maliciosos.

Nosso trabalho explora as características específicas do Interleave na proposta do mecanismo de incentivo, que tem aspectos que podem ser relacionados com mecanismos de reputação e reciprocidade, como discutido nestes surveys.

\section{AMBIENTE DE SIMULAÇÃO}

Para verificar o impacto da presença de nós não cooperativos, e posteriormente mensurar se o mecanismo de incentivo proposto pode garantir uma boa qualidade de vídeo, optou-se por criar as redes sobrepostas de teste em um simulador de rede P2P, o PeerSim [7], desenvolvido em Java, utilizando o módulo P4S [6]. Foi utilizado o modelo de simulação orientado a eventos, que inclui a camada de transporte para melhor retratar a realidade. $\mathrm{O}$ módulo $\mathrm{P} 4 \mathrm{~S}$ provê uma classe que simula o protocolo de difusão $\mathrm{Push} / \mathrm{Pull}$, e o gerenciamento de alocação de banda.

No simulador a rede sobreposta configurada é composta por um nó fonte que gera os pedaços a uma taxa constante. Os demais nós são inicializados ao mesmo tempo em modo passivo e seus vizinhos são escolhidos de forma aleatória e simétrica. Tal restrição faz parte do comportamento de um nó típico no protocolo Push/Pull.

Após a inicialização, o nó fonte envia pedaços para os seus vizinhos que, ao receber seu primeiro pedaço, são ativados e ficam aptos a solicitar e enviar pedaços, ativando então outros vizinhos que repetem o processo. Em um certo ponto espera-se que todos os nós estejam ativos. A simulação chega ao fim quando todos os nós possuírem todos os pedaços ou quando o tempo máximo de simulação for alcançado.

Dois tipos de vídeo foram utilizados nas simulações: de entretenimento, 5 minutos, obtido a partir da TV UOL, e educacional, 20 minutos, do servidor eTeach, Univ. Estes 
mesmos vídeos foram utilizados em [5]. Por questões de espaço os resultado serão apresentados apenas para o exemplo de vídeo educativo. Utilizamos também os mesmos parâmetros para cada um dos vídeos, resumidos na Tabela 1. Além disso, definiu-se a largura de banda de upload em $600 \mathrm{~kb} / \mathrm{s}$ para todos os nós, considerada compatível com as redes comerciais disponíveis [4], e o número mínimo de vizinhos de cada nó em 12, baseado nos resultados de [1], onde a rede tornava-se estável com valores de vizinhos maiores ou iguais a este.

TABELA I. PARÂMETROS DOS VÍDEOS UTILIZADOS

\begin{tabular}{|l|c|c|}
\hline Parâmetro & Entreten. & Educativo \\
\hline Tamanho do vídeo (pedaços) & 30 & 120 \\
\hline Tamanho do vídeo (minutos) & 5 & 20 \\
\hline Taxa de transmissão do vídeo & 350 & 350 \\
\hline Duração do pedaços de vídeo (s) & 10 & 10 \\
\hline Tamanho do pedaço do vídeo (kB) & 437,5 & 437,5 \\
\hline
\end{tabular}

Quatro métricas foram escolhidas para avaliar o desempenho durante as simulações:

1) Tempo Total: Instante de tempo no qual todos os pedaços foram recebidos por todos os nós da rede sobreposta.

2) Qualidade de Vídeo: Definida em [2] através da Equação 1, onde $T$ é a quantidade total de pedaços da distribuição de vídeo e $R$ o número de pedaços que chegaram antes do instante em que seriam utilizados para a reprodução do vídeo.

$$
Q=\frac{R}{T} \quad \text { (1) } \quad C=\frac{T_{r}-T_{e}}{T_{r}}
$$

3) Índice de Continuidade: Definido em [5] de acordo com a Equação 2, onde Te representa o tempo em que o vídeo fica congelado aguardando por pedaços atrasados e $T r$ o tempo total de reprodução. Quanto maior o valor, menor o tempo gasto com interrupções. Um índice igual a 1 é o desejável.

4) Atraso Inicial: Para esta métrica foi definida uma política simples: aguardar a chegada dos três primeiros pedaços, $(0,1$ e 2) para iniciar a reprodução. [4] apresenta mais detalhes sobre os problemas e efeitos de algumas formas para o cálculo desta métrica na distribuição de vídeo.

\section{IMPACTO DOS NÓS COOPERATIVOS NA REDE}

A avaliação dos nós não cooperativos considerou três modelos diferentes (ampliando o trabalho realizado em [4] que modelou apenas o $2^{\circ}$ tipo) alterando o comportamento original de um nó no protocolo Push/Pull nas seguintes variações:

Tipo 1: Nega explicitamente a requisição de um pedaço no modo pull, mas encaminha voluntariamente os pedaços para seus vizinhos durante o modo push.

Tipo 2: Responde a requisições por pedaços quando solicitado, mas não encaminha pedaços voluntariamente no modo push.

Tipo 3: Combina os comportamentos dos tipos anteriores e não colabora de forma alguma, apenas usufrui dos recursos.

Foram realizadas 150 rodadas de simulação para cada um dos 3 tipos de nós não cooperativos, transmitindo-se o fluxo de vídeo a partir de um nó fonte, em uma rede sobreposta com 200 nós. 15 diferentes sementes foram utilizadas com a porcentagem de nós não cooperativos na rede variando de $0 \%$ (nenhum nó não cooperativo), 5\%, 10\%, 15\% até 20\%. Os gráficos apresentam no eixo vertical as médias das métricas obtidas com um intervalo de confiança de $90 \%$, enquanto que o eixo horizontal representa as porcentagens de nós não cooperativos.
O desempenho da rede sobreposta na presença de nós Tipo 1, nas métricas de tempo total, índice de continuidade e qualidade do sistema não foram observadas diferenças significativas entre os nós cooperativos e não cooperativos (gráficos omitidos por limitação de espaço). No entanto observa-se que para a métrica de atraso inicial, os nós cooperativos conseguiram obter um resultado ligeiramente melhor do que os nós não cooperativos, ou seja, conseguiram receber os pedaços iniciais do vídeo primeiro.

Verifica-se que nós do Tipo 1 não afetam tanto a rede e não causam grande impacto no desempenho. Este resultado é compatível com a característica do estado pull ser usado menos que o estado push, apenas para preencher buracos na difusão dos pedaços, embora, intuitivamente, negar um pedaço de vídeo quando solicitado possa ser considerado o pior comportamento não cooperativo.

Com os nós do Tipo 2 (Figura 1) podemos observar em todas as métricas, um comportamento mais agressivo em comparação aos nós Tipo 1. Os valores obtidos para todas as métricas representam degradação de desempenho à medida que a quantidade de nós não cooperativos aumenta. Para o caso específico com $20 \%$ de nós não cooperativos, por exemplo, o aumento no atraso inicial chega a $28 \%$ em relação ao cenário em que estes nós não estão presentes.
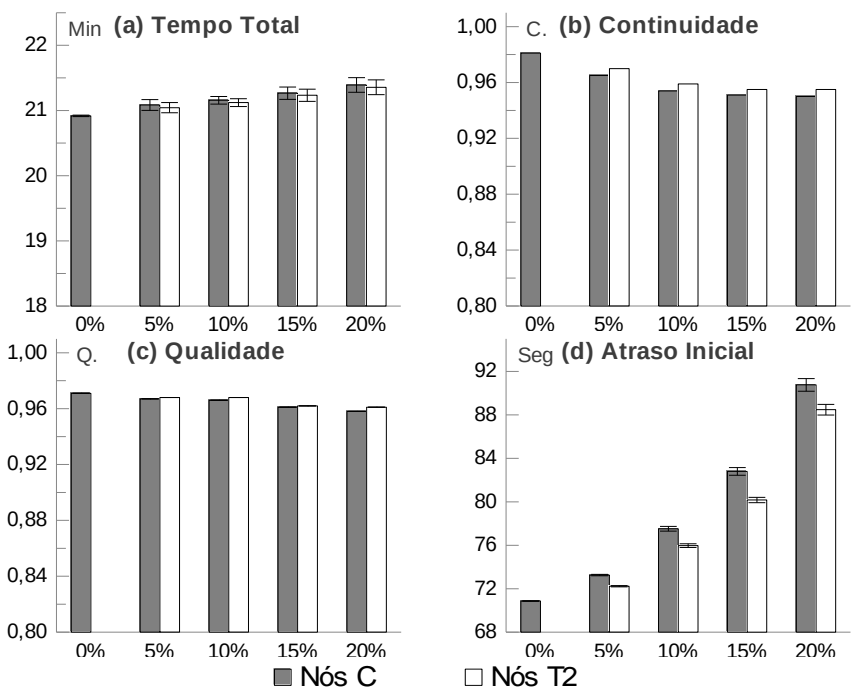

Fig. 1. Resultados para nós não cooperativos Tipo 2

Em geral, os nós não cooperativos obtiveram resultados ligeiramente melhores que os nós cooperativos. Nós do Tipo 2, ao contrário do tipo anterior, são nocivos a rede, degradando a distribuição de vídeo com atrasos na recepção de pedaços, congelamento da reprodução no cliente e maiores tempos de espera para iniciar e terminar a transmissão e reprodução.

O nó do Tipo 3 pode ser considerado o mais nocivo, unindo as características dos nós de Tipo 1 e 2 . Nas simulações (gráficos não apresentados) nota-se o tempo total cresce de forma expressiva de acordo com o aumento da quantidade de nós não cooperativos com uma variação muito grande para esta métrica. Nós próximos da fonte, apresentam valores melhores, enquanto outros podem estar cercados por nós Tipo 3, apresentando péssimo desempenho.

As outras métricas também apresentam resultados que refletem desempenho ruim. Por exemplo, o índice de continuidade é afetado na presença de apenas 5\% de nós Tipo 3. Em resumo, um nó Tipo 3 reflete um comportamento incompatível com o projeto das redes peer-to-peer, e sua 
ligação com a fonte ou com nós próximos a esta pode causar bloqueios na rede caso este nó esteja ligado a muitos nós cooperativos e obtenha pedaços de interesse de outros nós ligados a ele.

\section{MECANISMO DE INCENTIVO}

A constatação dos efeitos de nós não cooperativos induziram a proposta do mecanismo de seleção dinâmica de vizinhos. A abordagem é fazer com que cada nó avalie periodicamente o comportamento de seus vizinhos e substitua aqueles que estejam cooperando menos. Ao longo do tempo, nós com o mesmo nível de cooperação tendem a se agrupar, ainda que uma vizinhança com nós não cooperativos tenha sido inicialmente estabelecida. Com isso, nós muito cooperativos terão uma boa experiência no uso do sistema, enquanto que os menos cooperativos perceberão um serviço com qualidade inferior, incentivando-os a cooperarem mais.

\section{A. Cálculo das pontuações}

Mecanismos que utilizam a agregação de informações dos nós tem sido amplamente utilizados em redes $\mathrm{P} 2 \mathrm{P}$ privadas para compartilhamento de arquivos. Nestas redes, um tracker é responsável por armazenar informações sobre a quantidade de dados enviados e recebidos por usuários, e impõe a todos que mantenham a razão entre estes valores acima de um limiar [12]. Os autores de [9] mostram que esta política é efetiva para incentivar a colaboração dos usuários.

Para o mecanismo é proposta uma métrica que considera o número de pedaços de vídeo enviados e recebidos. Cada nó monitora a quantidade de pedaços recebidos e enviados de/para seus vizinhos em cada modo de transmissão. Periodicamente (sem sincronização com os nós da rede sobreposta) esta informação é enviada a um nó agregador, que a utiliza para consolidar a pontuação que classifica os nós na rede.

O número de pedaços recebidos por um nó $n$ via push é representado por $P_{R P u s h}(n)$ e o número de pedaços transmitidos via push por este mesmo nó é representado por $P_{T P u s h}(n)$. De forma análoga, o número de pedaços recebidos e transmitidos via pull por um nó n são representados por $P_{R P u l l}(n)$ e $P_{\text {TPull }}(n)$.

O cálculo proposto classifica cada nó no intervalo de valores entre 0 e 1 para cada um dos modos de transmissão (Eq. 3 e 4). Nós que fornecem maior quantidade de pedaços em comparação com o número de pedaços recebidos, em cada respectivo modo, tendem a obter uma pontuação mais próxima de 1 , sendo assim classificados como bons colaboradores.

$S_{\text {Push }}(n)=\frac{P_{\text {TPush }}(n)}{P_{\text {TPush }}(n)+P_{R \text { Push }}(n)}(3) S_{\text {Pull }}(n)=\frac{P_{\text {TPull }}(n)}{P_{\text {TPull }}(n)+P_{\text {RPull }}(n)}$

A Equação 5 consolida a pontuação de um nó em cada modo de transmissão em um único valor, considerando os resultados da Seção $\mathrm{V}$, que mostram que nós que não cooperam durante o estado push são mais prejudiciais à rede em comparação a nós que negam a transmissão de pedaços via pull. Pesos diferentes, $\mathrm{W}_{\text {Push }}$ e $\mathrm{W}_{\text {Pull, }}$, refletem a importância de dos modos push e pull na construção da pontuação.

$$
S(n)=\frac{S_{\text {Push }}(n) \times W_{\text {Push }}+S_{\text {Pull }}(n) \times W_{\text {Pull }}}{W_{\text {Push }}+W_{\text {Pull }}}
$$

Em testes preliminares no simulador verificou-se que em média $98 \%$ das entregas de pedaços são feitas através de push, e apenas $2 \%$ dos pedaços são enviados por requisições de pull, o que é compatível com os resultados apresentado na Seção V. Tais valores foram utilizados para o ajuste dos pesos durante a fase de execução das simulações.

$$
S_{\text {Final }}(n)=\frac{1}{1+\left|\frac{S(n)-1}{0,6}\right|^{6}}
$$

A fim de separar melhor os nós de acordo com suas características e facilitar a identificação do comportamento optou-se por aplicar a função sigmoide mostrada na Equação 6 nos resultados obtidos da Equação 5, obtendo-se então a pontuação final.

\section{B. Atualização das vizinhanças}

A etapa de atualização de vizinhanças também ocorre em periodicamente em cada nó da rede. Esta etapa é composta de duas fases: a remoção de vizinhos considerados não cooperativos, e a eventual adição de novos vizinhos para o nó.

Durante a fase de remoção, um nó solicita a pontuação de seus vizinhos ao nó agregador, e com estas informações, realiza para cada um destes uma avaliação do critério de remoção e, então mantém ou remove o vizinho.

Esta avaliação considera o módulo da diferença das pontuações, introduzindo uma faixa de tolerância. A faixa é definida para um nó $n$ por $S_{\text {Final }}(n) \pm k$ onde $k$ é o fator de tolerância, ou seja, a margem aceita por um nó para as pontuações de vizinhos em relação a sua. O fator de tolerância $k$ pode ser ajustado de forma a tornar nós mais tolerantes ou intolerantes a variações nas pontuações de seus vizinhos.

Após a etapa de remoções de vizinhos, um nó pode estabelecer conexões com outros nós para o preenchimento das ligações desfeitas na fase anterior. Para isto, o nó deve solicitar ao agregador uma lista de nós que participam da transmissão, e faz a seleção aleatória de novos vizinhos. Embora a escolha aleatória de um novo nó não garanta um vizinho cooperativo, ela simplifica a solução sem prejudicar a convergência no agrupamento de nós com características similares.

\section{Simulação E AVAliação dos Resultados}

A introdução do mecanismo no simulador é feita através da adição de um módulo de controle responsável por atuar como agregador das informações. No PeerSim, a adição de classes que utilizam a interface peersim.core. Control é a maneira recomendada para estender as funcionalidades do simulador de forma modular sem a necessidade de alterar diretamente outros artefatos de código já existentes.

O intervalo de atualização de vizinhanças utilizado foi de 30seg. Esse tempo é suficiente para que o mecanismo atue nos momentos iniciais da transmissão, buscando evitar principalmente que nós não cooperativos mantenham-se ligados à fonte nesta fase. $\mathrm{O}$ fator de tolerância foi ajustado para 0,4 , pois permite que nós cooperativos identifiquem nós não cooperativos fora de sua faixa de tolerância, e permite que outros nós permaneçam nessa faixa, assim como o nó fonte.

Os pesos atribuídos para $W_{\text {Push }}$ e $W_{\text {Pull }}$ foram 0,98 e 0,02 respectivamente, seguindo os resultados dos testes preliminares (Seções V e VI). As simulações do mecanismo dinâmico proposto seguem os mesmos parâmetros das demais simulações. Por questões de espaço apenas os resultados para os nós Tipo 2 são apresentados.

O tempo total da rede (Figura 2a) mostra que mesmo com o aumento da porcentagem de nós não cooperativos na rede, o mecanismo $(\mathrm{CM})$ proporcionou uma redução nos valores da métrica para nós cooperativos (C) em comparação às simulações sem o mecanismo (SM). Observa-se também que nós Tipo 2 (T2) tiveram um aumento no tempo total médio com a aplicação do mecanismo, funcionando como punição. 


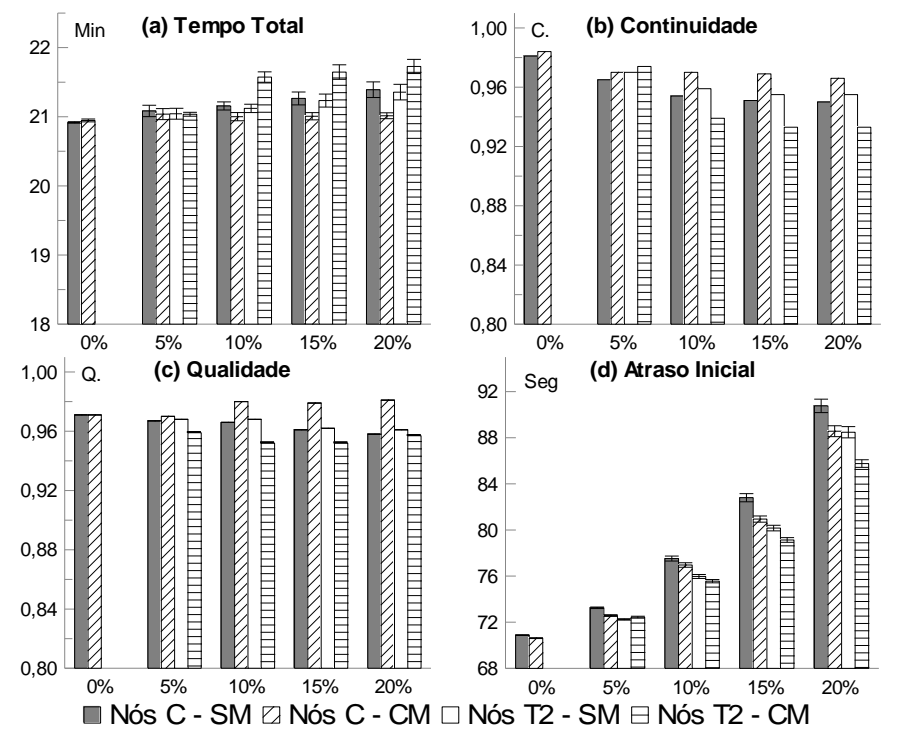

Fig. 2. Desempenho do mecanismo, para as 4 métricas, Nós Tipo 2

O índice de continuidade apresentou melhora para nós cooperativos (Figura 2b). Para o cenário com $20 \%$ de nós Tipo 2, o aumento no índice chegou a $10 \%$ em comparação a não utilização do mecanismo. Além disto, em geral os nós Tipo 2 obtiveram índices menores após a aplicação do mecanismo.

Nós cooperativos obtiveram um aumento na métrica de qualidade após a aplicação do mecanismo (Figura 2c). Além disto, o mecanismo elevou a qualidade obtida por estes nós à valores superiores aos encontrados em redes sem a presença de nós não cooperativos. Além disso, nós não cooperativos apresentaram redução na qualidade obtida. $\mathrm{O}$ atraso inicial foi reduzido para ambos os tipos de nós (Figura 2d).

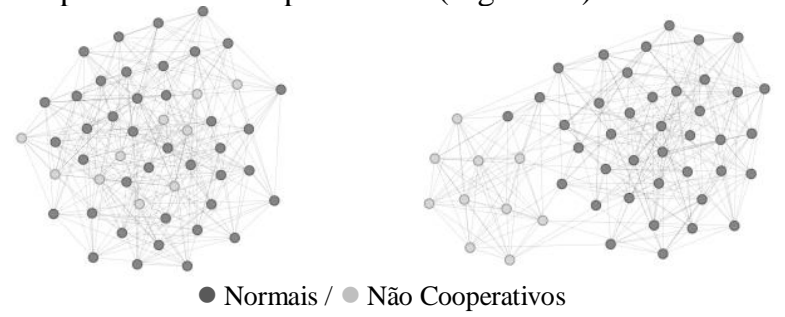

Fig. 3. Rede com 50 Nós, $20 \%$ Tipo 2, antes e depois do mecanismo

Com o mecanismo de incentivo observou-se uma melhora do desempenho da rede privilegiando os nós cooperativos em detrimento do desempenho dos nós não cooperativos. Podemos observar (Figura 3) que os resultados obtidos são consequência do agrupamento de nós com características semelhantes. Nós não cooperativos acabam estabelecendo um maior número de ligações entre si e com isso obtêm resultados inferiores.

\section{CONCLUSÕES}

O mecanismo dinâmico de seleção de vizinhos proposto, remove gradativamente as ligações de um nó com vizinhos não cooperativos e as substitui por novos vizinhos, desejavelmente cooperativos. Os resultados obtidos em simulação mostram que o mecanismo proporciona melhora para a rede como um todo nas várias métricas avaliadas para o serviço de transmissão de vídeo. Nós cooperativos foram beneficiados com qualidade de serviço superior à dos nós não cooperativos.

A proposta apresentada utiliza o número total de pedaços recebidos em cada estado, de cada vizinho $v$ para se identificar nós mais ou menos cooperativos. Em cada nó uma contagem separada dos pedaços recebidos de cada vizinho deve ser mantida e enviada periodicamente para o nó agregador. No simulador, para simplificar a implementação e facilitar a avaliação do mecanismo foram feitas simplificações: cada nó $v$ reporta o número total de pedaços enviados para os vizinhos.

Aspectos operacionais do mecanismo dinâmico estão sendo avaliados, como por exemplo, meios para detectar distorções (omissão ou erros propositais), que permitirão ao nó agregador consolidar as informações fornecidas por vizinhos, cooperativos ou não, a cerca do nó $n$ para construir uma pontuação consistente. Em trabalhos futuros, também será avaliado o uso de mecanismos de segurança em redes P2P para garantir a qualidade das informações e inibir falsificações, bem como outras formas de cálculo da pontuação.

Outro aspecto, a sobrecarga do protocolo para coordenar o mecanismo de incentivo é considerado um próximo passo, principalmente em relação ao churn, dado que a proposta inclui a realocação de nós na rede. Neste trabalho utilizamos os mecanismos disponíveis no PeerSim que permitem a reorganização da vizinhança, mas não nos permite medir o churn diretamente. Um ponto positivo em relação ao uso do protocolo Push/Pull é que a entrada de um novo nó na rede não requer que haja troca de mensagens para a sincronização de tabelas de pedaços disponíveis em cada vizinho. Por último, está sendo desenvolvido um protótipo de software para estudo, de modo a se analisar o desempenho da proposta em uma rede $\mathrm{P} 2 \mathrm{P}$ em condições reais.

\section{REFERÊNCIAS}

[1] CIGNO, R., RUSSO, A., CARRA, D. "On Some Fundamental Properties of P2P Push/Pull Protocols", HUT-ICCE 2008, pp. 67-73, Hoi an, Vietnam, Junho, 2008.

[2] HABIB, A., CHUANG, J., "Incentive mechanism for peer-to-peer media streaming”. IWQOS 2004, pp. 171-180, Montreal, Canadá, Junho, 2004.

[3] HALES, D. E., PATARIN, S. "Computacional sociology for system in the wild: the case of BitTorrent", IEEE Distribuetd Sys. Online, 2005.

[4] LIMA, F. M., SZTAJNBERG, A. "Análise de desempenho de redes p2p com protocol "Push/Pull" para distribuição de vídeo na presença de nós não cooperativos", SBRC 2011- Workshop P2P, Junho, 2011.

[5] MORAES, I.. "Distribuição de vídeo sobre redes par-a-par". 2009. Tese de Doutorado, COPPE Elétrica, UFRJ. Dezembro, 2009.

[6] "P2P 4 Streaming System", http://www.dit.unitn.it/networking/P4Smain.html. [09/2012].

[7] “A Peer-to-Peer Simulator”, http://peersim.sourceforge.net/. [09/2012]

[8] RAHMAN, M. R. "A Survey of Incentive Mechanisms in Peer-to-Peer Systems", TR CS-2009-22, Cheriton School of Computer Science, Univ. of Waterloo, 2009.

[9] ROSS, K.W., LIU, Z., et al., Understanding and Improving Incentives in Private P2P Communities, ICDCS 2010, Genova, Itália, 2010.

[10] SANGHAVI, B.S., el al.. "Gossiping with multiple messages". IEEE INFOCOM 2007. Dezembro, 2007.

[11] SAROIU, S., et al. "Measuring and analyzing the characteristics of Napster and Gnutella hosts". Multimedia Systems, No. 9, pp. 170-184, Springer, 2003

[12] SU, X., Dhaliwal, S.K., "Incentive Mechanisms in P2P Media Streaming Systems," Internet Computing, IEEE, vol.14, No.5, pp.74-81, 2010.

[13] TRAN, D. A., HUA, K. A., DO, T. T. "A Peer-to-Peer Architecture for Media Streaming”. IEEE JSAC. Vol. 22, No.1, pp. 121-133, 2004.

[14] XINYAN, Z., et al. "CoolStreaming/DONet: A Data-driven Overlay Network for Peer-to-Peer Live Media Streaming”. IEEE INFOCOM 2005. Vol. 3, pp. 2102-2111. Março, 2005.

[15] ZHANG, K. Antonopoulos, N., Mahmood, Z. "A Review of Incentive Mechanism in Peer-to-Peer Systems," 1st Intl. Conf.e on Advances in P2P Systems, pp. 45-50, 2009 